\title{
POTENTIEL ET LIMITES DES METHODES RADAR POUR LA CARTOGRAPHIE DU RELIEF EN AMAZONIE
}

\author{
Laurent Polidori \\ CESBIO, Université de Toulouse, CNES/CNRS/IRD/UPS, Toulouse, France \\ 18, avenue Edouard Belin - bpi 2801 - 31401 Toulouse Cedex 9 - France \\ laurent.polidori@cesbio.cnes.fr
}

\section{Résumé}

En raison des conditions climatiques, l'imagerie radar aérienne et spatiale est une source d'information essentielle pour la mise à jour de l'information topographique en Amazonie. Cet article présente un panorama des initiatives de cartographie de l'Amazonie au moyen de techniques radar en planimétrie ainsi qu'en altimétrie. Les tendances instrumentales et algorithmiques sont décrites. Les défis de la recherche sur les méthodes de cartographie radar et leurs applications sont discutées.

Mots-clés : imagerie radar, topographie, Amazonie

\begin{abstract}
Due to the weather conditions, airborne and spaceborne radar imagery is an essential source of information for updating the topographic information in the Amazon. This article presents an overview of mapping initiatives in the Amazon using radar techniques in planimetry as well as in altimetry. Instrumental and algorithmic tendencies are described. The challenges of research on radar mapping methods and their applications are discussed.
\end{abstract}

Keywords : radar imagery, topography, Amazon

\section{Introduction}

La cartographie du relief terrestre peut tirer parti de nombreuses techniques. Les relevés de terrain ont depuis longtemps cédé la place à la photogrammétrie, que toutes les agences de cartographie officielle mettent en œuvre à partir de prises de vue aériennes depuis la fin de la Seconde Guerre mondiale. Les principes de la photogrammétrie ont été étendus à l'imagerie spatiale dès que des acquisitions ont été possibles en configuration stéréoscopique (notamment depuis le lancement de SPOT-1 en 1986). La photogrammétrie permet d'obtenir un MNT (modèle numérique de terrain) qui décrit la géométrie du sol, ou un MNS (modèle numérique de surface) qui décrit la géométrie de l'enveloppe supérieure du sursol (arbres, bâtiments, ouvrages divers) (Kasser \& Egels 2002). D'autres techniques sont venues compléter la boîte à outils du cartographe, notamment le lidar (qui exploite les échos laser pour localiser la surface à partir d'acquisitions terrestres, aériennes ou spatiales) et le radar à synthèse d'ouverture, embarqué sur avion ou satellite, et dont les images, qui ont l'avantage d'être très peu affectées par les conditions météorologiques, sont extrêmement sensibles au relief, ce qui en permet la restitution par différentes méthodes: la radargrammétrie, la radarclinométrie et surtout l'interférométrie (Polidori 1997). A noter que l'altimétrie radar, représentée aujourd'hui par le satellite Jason-3 et que l'on n'évoquera pas au-delà de ce panorama introductif, a été conçue pour déterminer la topographie des océans avec une longueur d'onde spatiale de l'ordre du kilomètre, ce qui limite considérablement son intérêt pour la cartographie du relief continental.

En Amazonie, la couverture nuageuse a suscité depuis plusieurs décennies un fort intérêt pour les techniques radar. Depuis le projet RADAM qui a offert à l'Amazonie sa première couverture complète il y a près d'un demisiècle, toutes les innovations sur les capteurs radar comme sur les méthodes de traitement ont trouvé sur ce territoire ingrat un champ d'expérimentation unique. La cartographie de l'Amazonie n'a pas totalement rattrapé son retard pour autant, mais la supériorité du radar sur toutes les autres techniques n'a de cesse d'y être confirmée.

Cet article propose un état des lieux des initiatives de cartographie de l'Amazonie au moyen de techniques radar. Le contexte géographique et institutionnel est brièvement rappelé dans la section 2 . On expose ensuite les principales initiatives de cartographie 2D (section 3 ) et 3D (section 4). Ces initiatives sont confrontées aux besoins de cartographie du territoire amazonien et les promesses des solutions actuelles et futures sont évoquées (section 5).

\section{Contexte géographique}

L'Amazonie est une vaste plaine boisée qui s'étend entre la Cordillère des Andes et l'océan Atlantique, au nord du continent sud-américain (Droulers 1995). Le Brésil en occupe les deux tiers, le tiers restant se répartissant sur la périphérie du bassin versant de l'Amazone entre huit états limitrophes: Bolivie, Pérou, Equateur, Colombie, Venezuela, Guyana, Suriname et France (à travers la Guyane française). Au-delà du strict périmètre du bassin versant de l'Amazone, la région dite amazonienne s'étend aussi jusqu'au littoral des Guyanes, au nord du continent.

Le climat de l'Amazonie est chaud et humide et la couverture nuageuse est importante pendant la plus grande partie de l'année ce qui constitue un obstacle majeur pour la mise en œuvre de techniques de télédétection optique.

L'Amazone est le deuxième fleuve du monde par la longueur derrière le Nil, et le premier par le débit, représentant à l'embouchure $20 \%$ de l'eau douce transportée par la totalité des fleuves de la planète. Le relief est très modéré et couvert d'une forêt dense dont les arbres atteignent souvent $40 \mathrm{~m}$ de haut et qui masque 
le sol pour la plupart des capteurs de télédétection. Les modifications du paysage sont rapides: croissance urbaine, hydrographie, déforestation etc. Sur le plateau des Guyanes, l'évolution du littoral, véritable enjeu scientifique, est si rapide que la cartographie conventionnelle est impropre à fournir une description actualisée du trait de côte (Polidori 2008). Hormis les deux mégalopoles brésiliennes Belém et Manaus, capitales des états de Pará et Amazonas respectivement, la population se trouve dans des centres urbains de taille moyenne et dans des communautés villageoises dispersées.

Dans chacun des pays qui occupent une partie de l'Amazonie, la cartographie du territoire est confiée à une agence publique, civile ou militaire (à l'exception du Guyana et du Suriname où la cartographie est assurée par diverses institutions nationales ou étrangères, ou par des organismes privés): Instituto Geográfico Nacional (Pérou), Instituto Geográfico Militar (Equateur), Instituto Geográfico Agustín Codazzi (Colombie), Instituto Geográfico Militar (Bolivie), Instituto Geográfico Venezuela Simon Bolívar (Venezuela). La Guyane française étant un département français, sa cartographie fait partie des missions de l'IGN.

Au Brésil, la cartographie du territoire national est confiée à un institut fédéral, I'IBGE (Instituto Brasileiro de Geografia e Estatística, qui équivaut en France à la réunion de I'IGN et de I'INSEE). Toutefois, I'Armée de Terre (exército) a des prérogatives propres en matière de cartographie de l'Amazonie, d'une part parce que les expéditions géodésiques au cœur de la forêt amazonienne nécessitent des moyens que seule l'armée est en mesure de déployer, et d'autre part parce que la cartographie accompagne la mission stratégique d'occupation du territoire que les gouvernements successifs ont confiée à l'armée depuis plusieurs décennies sur l'ensemble de l'Amazonie et notamment le long des frontières internationales. Par ailleurs, I'INPE (Instituto Nacional de Pesquisas Espaciais) est chargé par le gouvernement fédéral du suivi par satellite de la déforestation amazonienne et produit des bilans cartographiques et statistiques de l'évolution du phénomène (INPE 2013).

Cependant, les agences gouvernementales qui ne maîtrisent pas toujours les technologies radar en font de ce fait un usage limité. Ainsi, l'Amazonie sert parfois de site d'expérimentation, dans le cadre de démonstrations de faisabilité qui ne cherchent pas forcément à s'intégrer dans les programmes nationaux de cartographie, voire à l'insu des agences publiques de cartographie.

\section{Les initiatives de cartographie 2D}

Le radar qui utilise des micro-ondes (entre $1 \mathrm{~cm}$ et $1 \mathrm{~m}$ ) peut fournir des images en subissant très peu d'impact de l'atmosphère. C'est pourquoi, compte tenu du climat de l'Amazonie, l'imagerie radar a été utilisée très tôt comme source d'information topographique.

A partir de 1970, le gouvernement brésilien commande une campagne radar aéroportée d'une étendue inédite (plus de 4,3 millions de $\mathrm{km}^{2}$ ) : le projet RADAM. Le radar en bande $X$ GEMS (Goodyear electronic mapping system) embarqué sur une Caravelle est un SLAR (radar aéroporté à visée latérale), le radar à synthèse d'ouverture n'étant pas arrivé à maturité à cette époque. II survole et cartographie en 1971 l'Amazonie légale (ensemble des 9 états brésiliens couvrant l'Amazonie). A l'issue de 1500 heures de vol, le produit obtenu est une mosaïque d'images analogiques au 1:400 000 avec un recouvrement de $25 \%$ et 45 points géodésiques sont déterminés avec le système Transit pour géoréférencer la mosaïque (Azevedo 1971, Van Roessel et Godoy 1974). On en présente deux exemples typiques sur la figure 1.

Pendant plusieurs années, des équipes scientifiques pluridisciplinaires sillonnent la forêt amazonienne pour y étudier les ressources naturelles grâce à cette nouvelle cartographie, et développent une compétence dans l'interprétation des images radar (figure 2). Le succès de cette campagne conduit le gouvernement brésilien à étendre la couverture radar à tout le territoire national en 1975. Au début des années 2000, l'intérêt pour ces données ne faiblissant pas, elles sont numérisées et subissent des traitements radiométriques, notamment une égalisation du diagramme d'antenne (Escobar et al. 2005). Les données sont disponibles gratuitement sur le site de la CPRM (service géologique du Brésil).
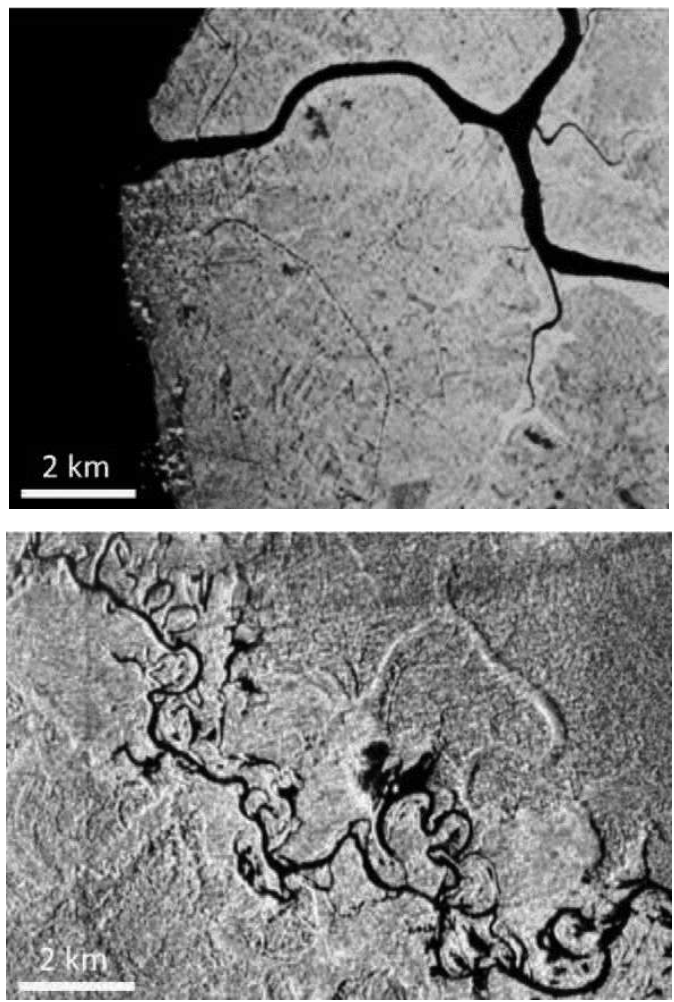

Figure 1 : images du projet RADAM (C CPRM) : le port d'lcoaraci sur la baie du Guajará (banlieue de Belém, Pará) en haut ; l'amont du rio Riorini (Amazonas) en bas

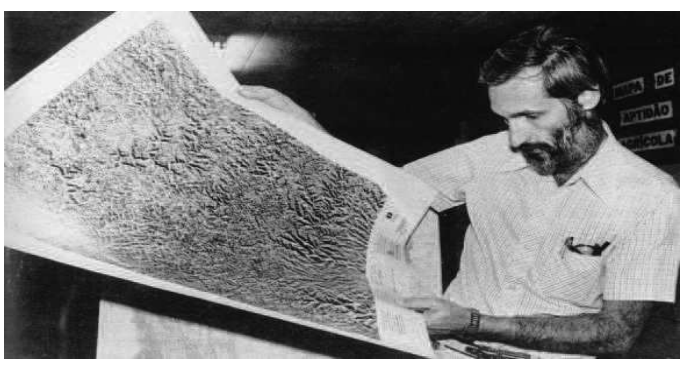

Figure 2 : utilisation des données du projet RADAM pour des études pédologiques en Amazonie @ INPE.

On assiste à des initiatives similaires dans les pays voisins. La figure 3 montre une mosaïque radar publiée par le ministère des mines et hydrocarbures au Venezuela. 
Cependant ces acquisitions sont généralement faites avec des instruments à très courte longueur d'onde (bandes $K$ et $X$ donc inférieures à $3 \mathrm{~cm}$ ) dont la propagation est susceptible d'être fortement perturbée en présence de pluies tropicales. Ce phénomène, également constaté dans les images du capteur X-SAR embarqué sur la navette spatiale en 1994 (figure 4), remet en cause l'idée un peu simpliste selon laquelle le radar serait un capteur « tout temps ».
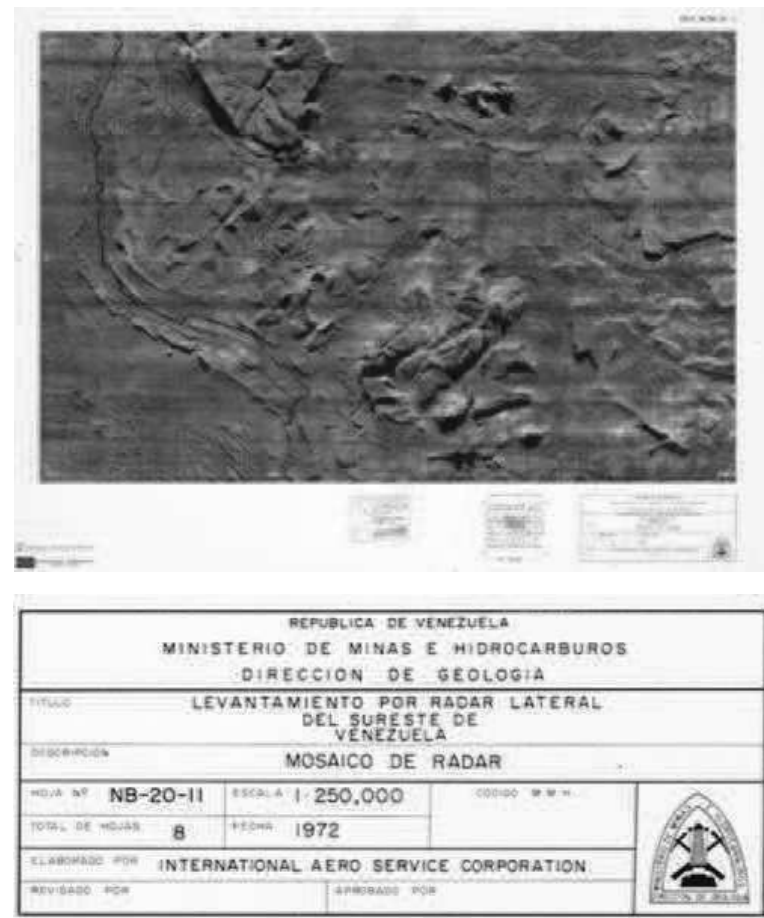

Figure 3: mosaïque radar pour la cartographie $d u$ territoire vénézuélien (1972) et détail du cartouche.

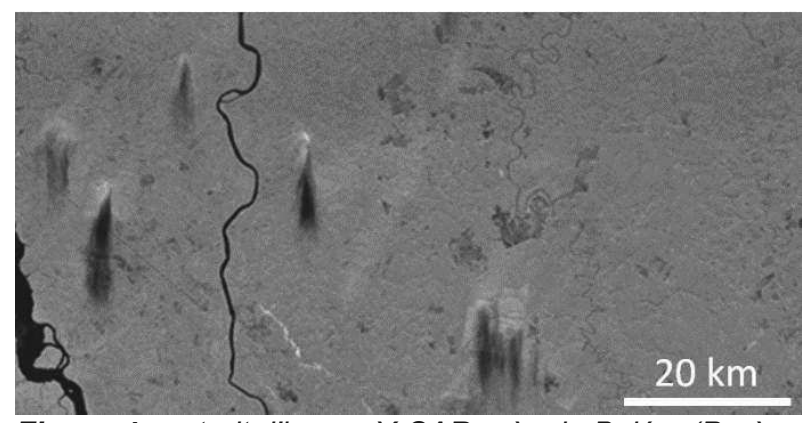

Figure 4 : extrait d'image $X-S A R$ près de Belém (Parà, Brésil) (C) DLR.

Dans les années 1990, notamment après le Sommet de la Terre à Rio de Janeiro, la classe politique et l'opinion internationale prennent conscience des enjeux écologiques et sociaux liés à l'Amazonie, et la protection de cette région devient un des objectifs majeurs de l'observation de la Terre. Un nouveau système spatial peut ainsi asseoir sa légitimité sur sa capacité à améliorer la cartographie de l'Amazonie, caractériser le milieu forestier et suivre la déforestation.

En 1992, dans le cadre d'une initiative conjointe du CCRS (Centre Canadien de Télédétection) et de la CIDA (Canadian International Development Agency), la campagne aéroportée SAREX (South American Radar Experiment) est organisée pour valoriser ERS-1 (satellite lancé en 1991 avec un radar en bande C) et préparer Radarsat (qui sera lancé en 1994). Des acquisitions radar aéroportées en bande $C$ sont réalisées avec le Convair
580 du CCRS dans six pays : Brésil, Venezuela, Guyana, Guyane française, Colombie et Costa Rica (Wooding et al. 1994). En Guyane française, ces données sont notamment exploitées pour l'étude des mangroves (Mougin el al. 1994). En Amazonie, il s'agit d'étudier le potentiel de l'imagerie radar pour l'étude des forêts tropicales dans le cadre de travaux qui seront capitalisés avec le programme GlobeSAR (Brown et al. 1996). Toutefois, le choix instrumental de la bande $C$, qui pénètre très peu dans la végétation restreint la portée de l'étude, et l'on sait depuis, que ce domaine de longueur d'onde est loin d'être le plus adapté aux environnements forestiers.

La même année, une autre démonstration est faite du potentiel du radar spatial pour la cartographie 2D des régions équatoriales avec le projet Guyana through the clouds (Rudant 1994): une couverture complète de la Guyane française (env. $84000 \mathrm{~km}^{2}$ ) avec une mosaïque d'images ERS-1. La communauté scientifique met immédiatement ces données à profit pour l'étude des milieux naturels. Toutefois, la mosaïque élaborée de manière artisanale n'a pas la qualité géométrique requise pour un véritable produit cartographique. Quelques années plus tard, l'IGN traite les mêmes données avec des méthodes de spatiotriangulation rigoureuses et édite des cartes au $1: 200.000$ (cf. extrait sur la figure 5) où les informations graphiques et toponymiques sont projetées sur fond d'image radar ortho-rectifiée. Dans des territoires où la couverture forestière est très homogène, le vert uniforme de la cartographie traditionnelle est ainsi remplacé par la radiométrie radar, sensible à la structure du couvert forestier et surtout au micro-relief. Dans cette région du bouclier des Guyanes, où le relief ancien est accidenté mais peu élevé, les parallaxes et les ombres portées sont très limitées, et l'image ortho-rectifiée sur l'ellipsoïde faute d'un MNT, sans pour autant fournir une information altimétrique quantitative, offre une description fine des formes du relief.

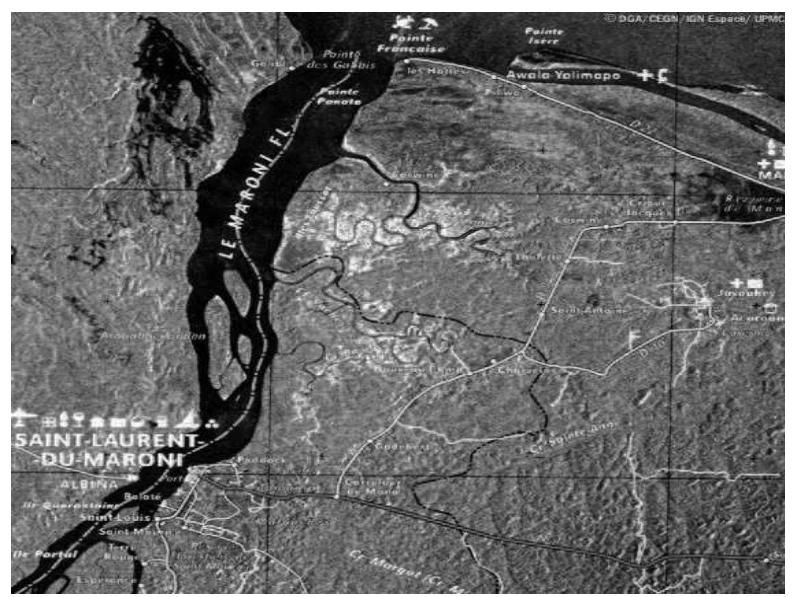

Figure 5: extrait de la carte IGN au $1: 200000$ de la Guyane française sur fond d'ortho-image ERS-1 @ IGN.

Dans les années 2000, des projets de recherche sur les écosystèmes amazoniens bénéficient de campagnes d'acquisition de données Radarsat dans le cadre de programmes visant à promouvoir ce satellite canadien. Dans le cadre du projet PIATAM-MAR financé par la société Petrobras, la communauté scientifique brésilienne, qui cherche à caractériser et cartographier la vulnérabilité des écosystèmes littoraux face au risque de pollution pétrolière, s'appuie sur une mosaïque d'images Radarsat qui s'étend de la frontière guyanaise jusqu'à la région de São Luis do Maranhão (Souza Filho et al. 
2009). Cependant, si ces images fournissent un fond de carte sans nuage où toutes les études peuvent être localisées, la bande $C$ se révèle peu adaptée à l'étude de ces écosystèmes. Ainsi, avec une pénétration très limitée dans le feuillage, il est difficile de distinguer différentes structures forestières, et de détecter les forêts inondées. De plus le contraste est très incertain entre l'eau et la terre ferme (comme le montre la figure 6), comme entre les zones boisées et déboisées.

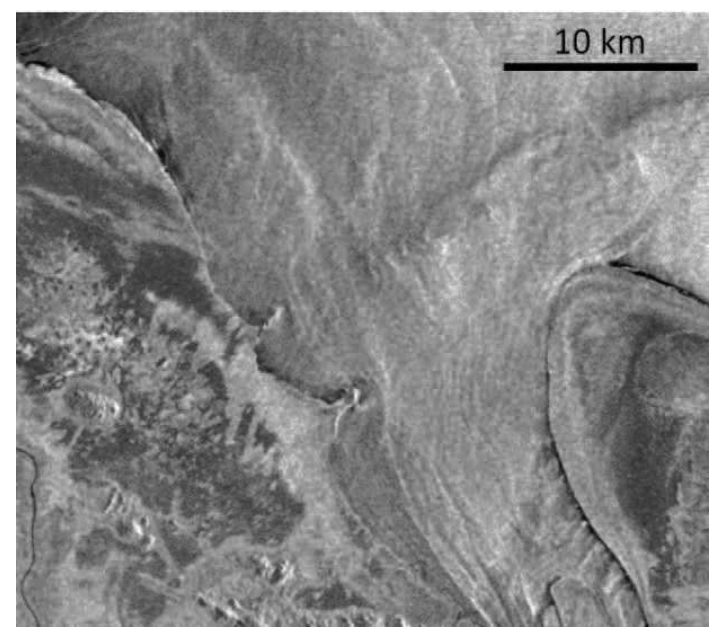

Figure 6: extrait d'image ERS-1 sur l'estuaire de l'Oyapock @ ESA.

Au contraire, les images en bande $L$ (longueur d'onde d'environ $25 \mathrm{~cm}$ ) apportent beaucoup d'information sur la déforestation et l'hydrographie. Ce qui explique le succès de la mosaïque élaborée dans toutes les régions tropicales à partir des images du satellite japonais JERS1 (figure 7, Siqueira et al. 2000).
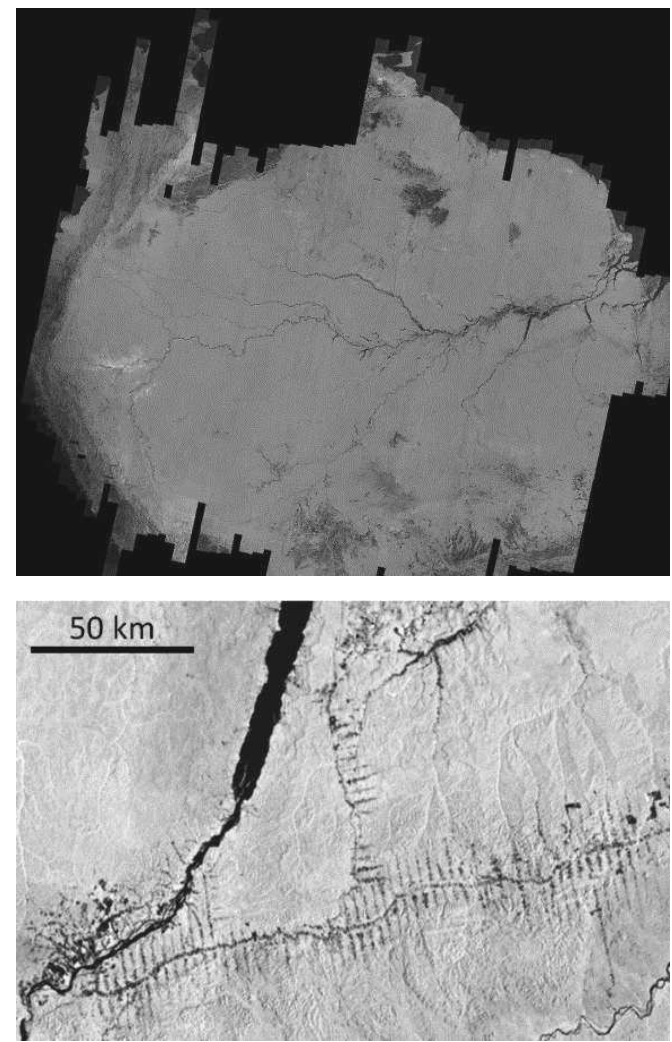

Figure 7 : mosaïque d'images radar en bande $L d u$ satellite japonais JERS-1, et extrait dans l'ouest du Pará (rio Tapajós et transamazonienne) @ NASDA/MITI.
Près de 30 ans après le programme RADAM qui visait à une meilleure connaissance du territoire, le gouvernement brésilien se dote de nouveaux moyens de surveillance du territoire en créant le SIVAM (Sistema de Vigilância da Amazônia) avec des capacités de contrôle de l'espace aérien et de cartographie radar aéroportée en bandes X et L (Silva 1998).

Aujourd'hui et sous réserve que les capacités de traitement suivent, c'est clairement la mission Sentinel-1 qui offre la promesse la plus crédible d'une connaissance fiable et actualisée du territoire amazonien. Les limites de la bande $C$ pour l'Amazonie ont été rappelées plus haut, toutefois la sensibilité de la polarisation croisée HV à la végétation et la cadence élevée de revisite, de 6 jours seulement si l'on utilise les deux satellites Sentinel-1, compensent en partie la sensibilité limitée de la bande $C$. Une série d'images géométriquement superposées (que l'on peut par exemple représenter sous la forme d'une composition colorée multidate comme celle de la figure 8) permet non seulement de détecter mais aussi de dater approximativement la déforestation. Si cette possibilité a donné lieu à des démonstrations spectaculaires en Amazonie, elle dépend néanmoins des conditions météorologiques et de l'état de la parcelle déboisée, le contraste pouvant être insuffisant en saison des pluies et lorsque les arbres coupés ont été laissés sur place (Le Toan et al. 2017).

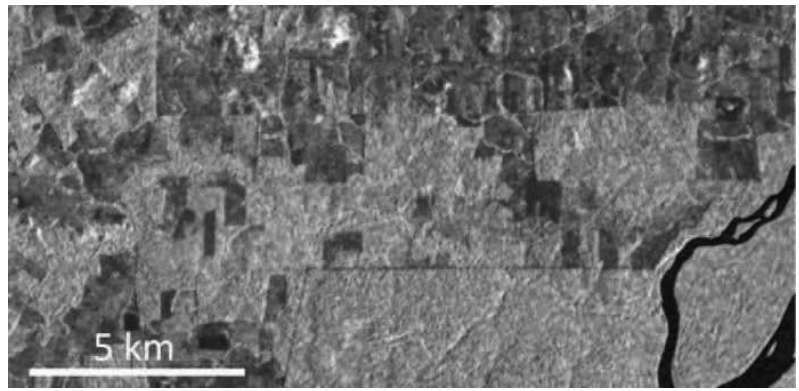

Figure 8: composition colorée multidate d'images Sentinel-1 en Amazonie brésilienne (rouge : 15/08/2016; vert : 02/10/2016 ; bleu : 14/10/2016) d'après Le Toan et al. (2017).

\section{Les initiatives de cartographie 3D}

La cartographie tridimensionnelle du relief des terres émergées est principalement réalisée par photogrammétrie aérienne depuis la seconde moitié du $X X^{\text {ème }}$ siècle, une méthode complétée par la photogrammétrie spatiale avec les missions SPOT et ASTER notamment. Cependant, la couverture nuageuse qui caractérise les régions équatoriales donne aux techniques radar tout leur intérêt, et les images radar acquises sur l'Amazonie ont souvent servi à en tester des évolutions algorithmiques. Au-delà des conditions météorologiques qui limitent les observations optiques, toutes les méthodes de cartographie du relief sont potentiellement contraintes en Amazonie par les difficultés d'accès qui limitent la possibilité de matérialiser des points d'appui, et par la couverture forestière dont l'épaisseur, souvent supérieure à 30 mètres, ne peut être négligée par rapport aux variations altimétriques d'une topographie peu accidentée.

Trois techniques, tirant parti de la sensibilité au relief des images du radar à synthèse d'ouverture, ont été proposées depuis longtemps pour élaborer des MNT à partir de ces données: la radargrammétrie, la radarclinométrie et l'interférométrie. Dans les années 
1970-80, les principes théoriques de ces techniques sont maîtrisés et leur faisabilité est démontrée à partir de campagnes aéroportées et de missions de la navette spatiale notamment (Leberl 1990, Polidori 1991). Le lancement d'ERS-1 fin 1991 permet de disposer de grandes quantités d'images, et donc de perfectionner les méthodes de cartographie radar et de mieux en connaître le potentiel et les limites, les lancements de capteurs radar se poursuivant ensuite à un rythme soutenu avec notamment Radarsat et la mission tandem ERS-1/ERS-2.

La radargrammétrie étend le principe de la photogrammétrie à la géométrie radar, en calculant l'altitude à partir des parallaxes entre les images d'un couple stéréoscopique. Bien qu'étant une technique géométriquement rigoureuse, elle est limitée par la corrélation automatique à laquelle les images d'un capteur actif se prêtent difficilement. En effet, la radiométrie dépend de la position du capteur, qui varie nécessairement entre les deux acquisitions d'un couple stéréoscopique. Des tests réalisés sur la forêt guyanaise avec des données ERS (figure 9) ont montré que la précision était au mieux de l'ordre de la vingtaine de mètres (Vasseur 2002).

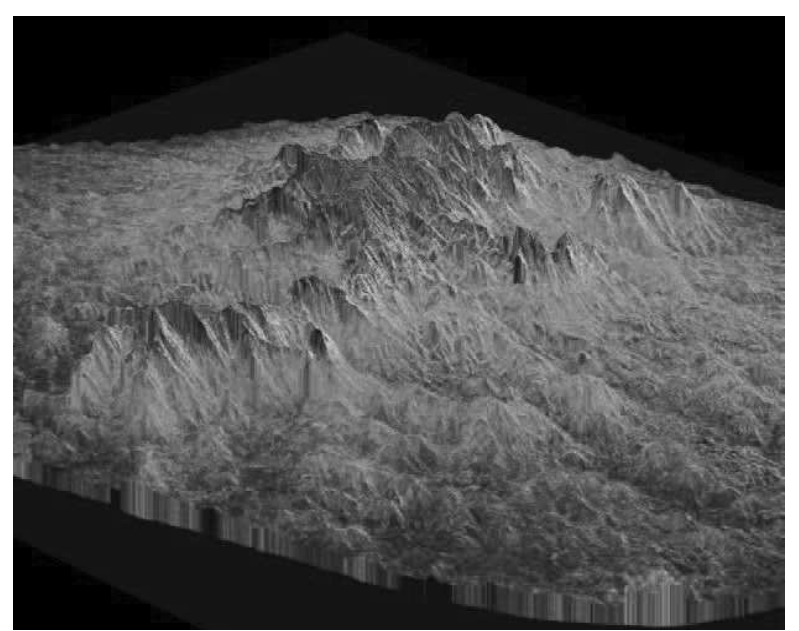

Figure 9: MNT radargrammétrique sur la Montagne de Kaw, Guyane française (d'après Vasseur 2002).

Le mode fin du système Radarsat a permis d'étendre les possibilités de la radargrammétrie, grâce à une résolution plus fine et à la possibilité de choisir parmi une gamme plus étendue d'angles de visée. Ainsi Toutin \& Amaral (2000) ont appliqué la radargrammétrie en Amazonie brésilienne pour évaluer la hauteur de la forêt sur les limites de parcelles déforestées, et la comparaison de leurs résultats avec des données in situ fait état d'une précision de $5 \mathrm{~m}$, ce qui offre une alternative intéressante à la photogrammétrie dans les régions nuageuses.

La radarclinométrie applique le principe du « shape from shading " pour estimer l'orientation du terrain à partir de la radiométrie de l'image radar. Cette technique, qui consiste à attribuer les variations d'intensité aux variations de pente, a l'avantage de pouvoir se contenter d'une seule image, mais elle est fortement dépendante des hypothèses qu'il faut injecter dans l'algorithme pour résoudre à deux reprises une équation à deux inconnues:

- pour déterminer une orientation dans l'espace (en deux dimensions, par exemple les pentes est-ouest et nordsud) à partir du seul angle d'incidence local (angle entre l'axe de visée supposé connu et le vecteur normal à la surface, inconnu);
- pour séparer dans la radiométrie radar les influences du coefficient de rétrodiffusion (lié aux caractéristiques du couvert) et de l'angle d'incidence local (lié à l'orientation du terrain), afin de pouvoir inverser un modèle de rétrodiffusion.

Aux contraintes liées à ces hypothèses s'ajoute l'inévitable propagation de l'erreur altimétrique lors de l'intégration de la pente.

Pour toutes ces raisons, la radarclinométrie n'est pas adaptée aux exigences classiques de la cartographie. Toutefois, la région amazonienne qui est en grande partie couverte de forêt permet raisonnablement de supposer constant le coefficient de rétrodiffusion et donc d'attribuer les gradients radiométriques aux seuls changements de pente. Ainsi des tests réalisés sur la forêt guyanaise à partir d'images ERS-1 font état d'une précision altimétrique de l'ordre de $20 \mathrm{~m}$ (Paquerault 1998). Les MNT obtenus sont donc peu précis en altimétrie, mais le fait que le calcul utilise la radiométrie comme indicatrice de la pente les rend relativement fidèles aux formes (figure 10).
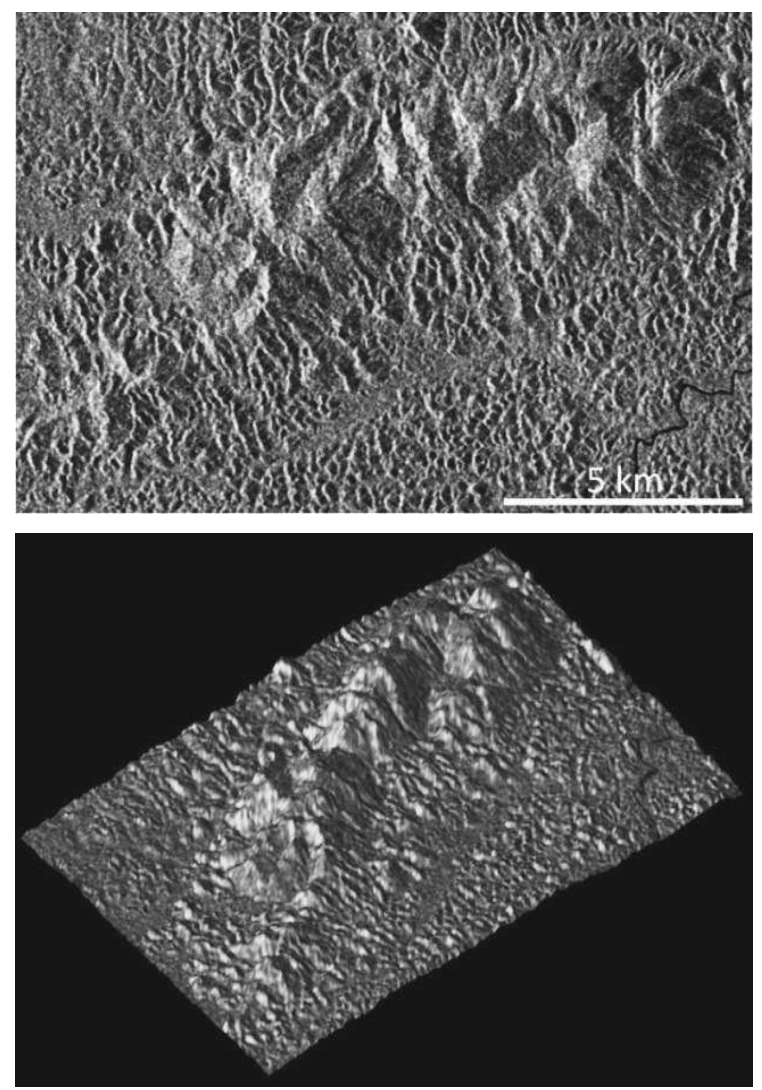

Figure 10 : image ERS-1 Montagne Plomb (Guyane) en haut ; MNT restitué par radarclinométrie habillé de l'image radar en bas (d'après Paquerault 1998).

L'interférométrie radar est de loin la plus performante des trois techniques. Elle consiste à déterminer l'altitude du terrain à partir de la différence de phase entre deux échos radar acquis depuis deux positions très proches de l'antenne. Le potentiel de cette technique pour la cartographie du relief à partir d'un couple d'images spatiales a été mis en évidence dans les années 1980 (Zebker \& Goldstein 1986) puis surtout après le lancement du satellite ERS-1 (Massonnet \& Rabaute 1993). Idéalement les deux images devraient être obtenues à partir de deux antennes lors d'un seul passage, toutefois la plupart des systèmes radar spatiaux disposent d'une seule antenne et doivent donc survoler 
deux fois la même zone pour produire un couple interférométrique, ce qui les expose à deux principales contraintes :

- tout d'abord la précision altimétrique dépend de la base stéréoscopique (espacement entre les deux orbites), qui n'est pas connue avant l'acquisition ce qui interdit une connaissance préalable de la précision du MNT obtenu ;

- par ailleurs et notamment en région amazonienne, les variations des conditions atmosphériques peuvent causer un déphasage important et donc une erreur d'altitude, et le mouvement aléatoire des feuillages peut causer une décorrélation de la phase qui limite la précision altimétrique, voire la faisabilité de la méthode.

L'interférométrie différentielle, qui nécessite des images acquises à des dates différentes pour mettre en évidence des mouvements de terrain, est sujette aux mêmes contraintes: impossibilité de prédire la précision et interférences de la forêt et de l'atmosphère (Ramos et al. 2012).

L'interférométrie à deux antennes permet de surmonter tous ces obstacles pour la cartographie du relief. Des expérimentations en mode aéroporté ont permis de valider le concept dans les années 1990 et ont conduit à la définition de la mission SRTM (Shuttle Radar Topography Mission). Après un vol de 11 jours de la navette Endeavour en février 2000, le traitement des couples interférométriques issus de cette mission a permis d'élaborer un MNT distribué au pas de $90 \mathrm{~m}$ sur l'ensemble des régions continentales tropicales et tempérées (Farr \& Kobrick 2001). En Amazonie, où le relief était par endroit moins bien connu que sur la planète Vénus, SRTM est devenu la référence en matière altimétrique et a donné lieu à de nombreuses applications en sciences de la Terre et de l'environnement (voir par exemple Mantelli et al. 2009, Hayakawa et al. 2010 etc.)

Rodriguez et al. (2006) évaluent la précision altimétrique de SRTM en s'appuyant sur des points de contrôle et obtiennent pour l'Amérique du sud un biais de 1,7 m et un écart-typpe de $4,1 \mathrm{~m}$ et des résultats comparables voire meilleurs sur d'autres continents. Mais ces résultats basés sur des données de terrain sélectionnées manuellement dans des secteurs favorables (en évitant notamment les constructions et les arbres) surestiment largement la qualité du produit. La plupart des études font plutôt état d'erreurs altimétriques absolues de l'ordre de la dizaine de mètres, inférieures à la spécification de 16 m (Bamler 1999).

Au Brésil, pour répondre à la demande d'une cartographie altimétrique un peu plus résolue, I'INPE (Institut Nacional de Pesquisas Espaciais) a entrepris de produire une base de données altimétriques au pas de $30 \mathrm{~m}$ par densification du MNT SRTM. Baptisée Topodata, cette base de données a été obtenue par krigeage avec un ajustement empirique des paramètres sur la base d'une expertise géomorphologique (Valeriano \& Rossetti 2012).

Toutefois, si SRTM - distribué par la suite avec une maille de $30 \mathrm{~m}$ - et le produit dérivé Topodata ont permis d'obtenir une cartographie du relief amazonien avec une complétude et une précision inédites, il ne s'agit pas à proprement parler de modèles numériques de terrain, car les ondes radar en bande $C$ pénètrent très peu dans le feuillage et le modèle 3D obtenu est donc beaucoup plus proche de la canopée que du sol (Dubois-Fernandez \& Koleck 2014). Face à cette limite des méthodes radar à courte longueur d'onde, le potentiel de la bande $P$ (longueurs d'onde proches du mètre) a été depuis longtemps exploré pour la cartographie du relief dans les régions boisées. Moreira et al. (2001) démontrent le potentiel d'une acquisition simultanée d'images en bande $X$ et $P$ en Amazonie mais seulement par un traitement interférométrique classique c'est-à-dire sans tirer parti de la polarimétrie.

Au Brésil, l'armée de terre a entrepris un programme de cartographie baptisé "Radiografia da Amazônia » destiné à couvrir l'Amazonie à partir d'acquisitions radar aéroportées en bandes $X$ et $P$, le traitement interférométrique conduisant à un MNT pour la bande $P$ et un MNS pour la bande $X$ (Correia 2011). La figure 11 montre un profil de $25 \mathrm{~km}$ de longueur extrait de ces deux produits. La différence entre les deux profils est de l'ordre de $25 \mathrm{~m}$ alors que la hauteur de la canopée se situe généralement entre 30 et $40 \mathrm{~m}$, ce qui conduit à considérer avec prudence l'hypothèse selon laquelle la bande $\mathrm{X}$ décrit le sommet de la canopée et la bande $\mathrm{P}$ décrit le sol.

La possibilité d'implémenter un radar imageur en bande $P$ sur un satellite est étudiée depuis les années 1990 (Chandra et Hounam 1998). La première mission radar en bande $P$ décidée par une agence spatiale est la mission Biomass de l'ESA (Le Toan et al. 2011). Destinée à la cartographie de la biomasse aérienne, cette mission devrait aussi permettre la cartographie du relief y compris à travers la forêt.

Parallèlement aux développements instrumentaux qui ont conduit à maîtriser la bande $\mathrm{P}$ en vue d'applications aux régions boisées, des techniques ont été proposées dans les années 2000 pour tirer le meilleur parti des signaux radar interférométriques sur des couverts volumiques. La technique PollnSAR combine les possibilités de la polarimétrie qui est sensible à l'organisation volumique du couvert et de l'interférométrie qui exploite la mesure de phase pour extraire des informations géométriques (Papathanassiou et al. 2000). Cette technique permet, lorsque les caractéristiques de l'onde radar et du milieu conduisent à un mélange d'effets de rétrodiffusion volumique et surfacique, d'estimer la hauteur du sol et de la canopée.

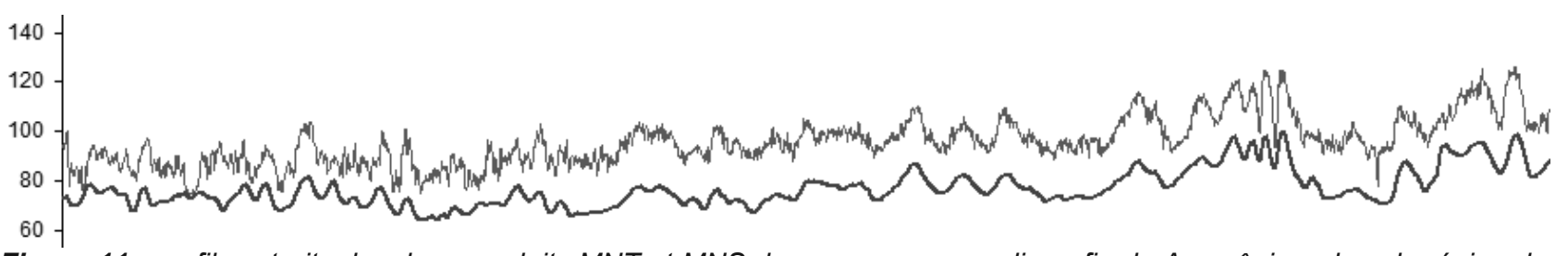

Figure 11 : profils extraits des deux produits MNT et MNS du programme " radiografia da Amazônia " dans la région de São Gabriel da Cachoeira (Brésil). 
La tomographie va plus loin encore. Cette technique déjà appliquée à l'imagerie médicale pour reconstituer des objets 3D, peut s'appliquer à des séries d'images radar acquises avec des angles d'incidence multiples sur des couverts forestiers comme la forêt, pour séparer par tranches de hauteurs les différentes contributions à l'intensité du signal rétrodiffusé (Reigber \& Moreira 2000). Cette technique qui a fait l'objet de démonstrations prometteuses avec des données en bande $L$, devrait permettre avec le développement de la bande $P$ d'acquérir des informations précieuses non seulement sur la topographie du sol et de la canopée, mais aussi sur la structure tridimensionnelle des couverts forestiers.

\section{Discussion}

\subsection{Les limites des méthodes radar en Amazonie}

Les sections précédentes ont évoqué la variété des méthodes de cartographie radar, tant $2 \mathrm{D}$ que $3 \mathrm{D}$, qui ont été appliquées avec plus ou moins de succès à l'Amazonie, soit pour une meilleure connaissance du territoire, soit pour confronter les capteurs et les algorithmes à cet environnement particulièrement ingrat. Les études visant à évaluer les différentes méthodes de cartographie au moyen d'images radar en ont montré les limites, liées soit aux choix instrumentaux (nous avons par exemple évoqué plus haut les limites de la bande $\mathrm{C}$ ), soit aux conditions environnementales, notamment météorologiques. Pour ne rappeler que trois exemples cités dans les sections précédentes, on observe notamment: (1) des taches dans les images en bandes $\mathrm{K} / \mathrm{X}$ en cas de forte pluie, (2) un déphasage produisant des erreurs dans les produits interférométriques, (3) une difficulté accrue de détection de la déforestation en saison des pluies. Ainsi la notion de capteur « tout temps » vient buter sur ses limites en Amazonie.

Malgré les promesses des méthodes radar, on est donc loin d'avoir trouvé la méthode idoine (choix des meilleures caractéristiques instrumentales et conditions environnementales favorables) pour la cartographie des régions boisées sous un climat tropical humide, par exemple en Amazonie. C'est particulièrement vrai pour la cartographie du relief. En effet, dans la majeure partie du bassin amazonien, la surface topographique a des amplitudes altimétriques qui n'excèdent guère la barre d'erreur des techniques mises en œuvre, et elle est masquée par un épais couvert forestier. Or, les techniques usuelles (photogrammétrie et techniques radar à courte longueur d'onde) donnent accès au MNS et non au MNT. Dans ce contexte, plusieurs questions de recherche restent ouvertes et nous pouvons en citer trois : comment obtenir un MNT vrai ? le MNS (plus facile à obtenir) peut-il se substituer au MNT ? la connaissance à priori des propriétés générales du relief terrestre peutelle compenser les limites des méthodes de mesure? Ces trois questions sont soulevées ci-après.

\subsection{Comment obtenir un MNT vrai ?}

Comment obtenir en présence de forêt un MNT vrai, c'està-dire un modèle numérique de la surface du sol, ou de l'eau en cas d'inondation permanente?

Depuis les années 2000 , les altimètres laser aéroportés fonctionnent avec un mécanisme de balayage latéral à très haute cadence qui permet d'acquérir des nuages de points très denses (plusieurs points par mètre carré), et l'instrumentation de mesure de position et d'attitude disponible sur le marché permet des précisions de l'ordre du décimètre. En forêt, une certaine proportion des impulsions laser atteint le sol à la faveur de trouées dans le feuillage. Cependant, pour une densité de feuillage donnée, la densité des points du sol n'est exploitable pour élaborer un MNT que dans des conditions de vol particulières, typiquement un vol lent et bas, proche de la canopée. Ainsi, même si l'altimétrie laser aéroportée est aujourd'hui la méthode la plus performante pour la cartographie du relief en région boisée, ces contraintes opérationnelles en limitent la viabilité économique pour de trop grandes étendues.

On a vu également que le radar en bande $\mathrm{P}$ offrait la possibilité d'accéder au sol pour peu que l'on soit capable de localiser le sol. Ce qui nécessite de connaître le comportement du signal radar dans sa traversée du couvert forestier ainsi que les résultats des techniques de traitement utilisées (interférométrie classique, PollnSAR ou tomographie). Les études menées dans le cadre de la préparation de la mission spatiale Biomass devraient permettre d'affiner les méthodes d'extraction du relief sous couvert forestier et d'évaluer l'incertitude d'une telle méthode. On doit s'attendre à ce que les performances dépendent des conditions environnementales (pouvant conditionner l'humidité du couvert) et de la pente.

Malgré le potentiel de l'altimétrie laser aéroportée et du radar en bande $\mathrm{P}$, le MNT obtenu à travers la forêt est forcément dégradé par rapport à ce que l'on obtiendrait en terrain dégagé. Un MNS étant plus facile à obtenir et d'ailleurs souvent déjà disponible, on peut être tenté de le substituer au MNT lorsque celui-ci fait défaut.

\subsection{Le MNS peut-il se substituer au MNT ?}

La plupart des applications des modèles altimétriques ont besoin de l'altitude du sol (MNT) et non du sursol (MNS). Or la plupart des méthodes de cartographie 3D fournissent un MNS plutôt qu'un MNT (ainsi le modèle SRTM qui est largement utilisé dans toutes les régions tempérées et tropicales et notamment en Amazonie, est en première approximation un modèle numérique de surface). II est donc intéressant de se demander dans quelle mesure et pour quels usages on peut utiliser un modèle numérique de la canopée pour caractériser le relief en vue d'applications hydrologiques et géomorphologiques. Si la grandeur recherchée est l'altitude, on peut considérer que l'erreur commise en utilisant le MNS en lieu et place du MNT se comporte comme un biais constant égal à la hauteur moyenne des arbres, que l'on peut retrancher au MNS pour " descendre » au niveau du sol, méthode couramment employée dans la production traditionnelle de cartes à moyenne échelle. Toutefois, la plupart des usages des MNT portent non pas sur l'altitude, mais sur des grandeurs dérivées comme la pente, l'aspect, la courbure.

La comparaison des profils altimétriques issus d'acquisitions aéroportées en bandes $X$ et $P$ dans le cadre du programme « radiografia da Amazônia » montre que les pentes du sol et de la canopée sont de plus en plus corrélées à mesure que le pas d'échantillonnage augmente. Ainsi, sur un site amazonien typique, l'erreur relative commise en confondant la pente du MNT et celle du MNS est de l'ordre de $5^{\circ}$ sur une distance de $30 \mathrm{~m}$ et de $2^{\circ}$ sur une distance de $90 \mathrm{~m}$ (Polidori et Simonetto 2014). 
L'idée du produit Topodata cité plus haut est intéressante dans ce contexte. En effet, il s'appuie sur le produit SRTM, initialement dégradé à $90 \mathrm{~m}$, donc peu influencé par la texture de la canopée, qu'il suréchantillonne à 30 $\mathrm{m}$ selon des critères exclusivement géomorphologiques. II est donc débarrassé des effets texturaux liés à la géométrie de la canopée, qui auraient dégradé la précision des pentes, d'où une meilleure qualité selon des critères géomorphologiques (Polidori et al. 2014).

5.4. La connaissance à priori des propriétés du relief terrestre peut-elle compenser les limites des méthodes de mesure?

Les deux questions précédentes s'intéressent à la possibilité de connaître la géométrie du sol au moyen d'une mesure nécessairement très grossière en raison de la présence du masque forestier (5.2) ou d'une mesure beaucoup plus précise mais décrivant en réalité la géométrie de la canopée et pouvant être utilisée abusivement comme une description de la topographie du sol (5.3). Dans les deux cas, on est en présence d'un MNT très imparfait. Toutefois, cette donnée est obtenue sans tenir compte des propriétés géomorphologiques du relief terrestre, ce qui laisse envisager des possibilités d'amélioration. La vérification de ces propriétés permet d'ajouter au contrôle usuel de précision géométrique des critères de qualité de nature géomorphologique (Polidori 1995, El Hage 2012). Le non-respect de ces propriétés permet de mettre en évidence un relief impossible (par exemple un profil de talweg présentant des remontées) ou seulement improbable (abondance de pentes très abruptes, incohérence du réseau hydrographique, anisotropies inexpliquées etc.). Les possibilités de contrôle s'en trouvent étendues, du moins pour les reliefs façonnés par le ruissellement de l'eau et donc structurés en bassins versants (Polidori et al. 2014). En revanche, la possibilité d'injecter ces propriétés pour contraindre le calcul du MNT reste un champ de recherche peu avancé.

\section{Conclusion}

Les techniques basées sur l'imagerie radar aéroportée ou spatiale ont permis une amélioration considérable de la connaissance du territoire amazonien. Tandis que de nouvelles campagnes d'acquisitions aéroportées et la conception de nouveaux capteurs spatiaux enrichissent progressivement la cartographie de cette région, les limites des techniques de cartographie 2D et $3 D$ se précisent et soulèvent des questions de recherche. Ces questions relèvent aussi bien des aspects méthodologiques liés aux instruments et aux algorithmes, que des aspects écologiques et géomorphologiques liés à l'organisation spatiale des bassins versants. Si ces deux domaines sont étudiés conjointement ils devraient permettre d'améliorer à la fois la qualité des produits cartographiques dont la gestion du territoire amazonien a besoin, et la compréhension des phénomènes naturels et anthropiques qui ont contribué à façonner cet écosystème et n'ont de cesse de le transformer.

\section{Références}

Azevedo L. (1971) Radar in the Amazon, Proceedings of the 7th International Symposium on Remote Sensing of Environment, vol. III, pp. 2303-2306.

Bamler R. (1999) The SRTM mission: a world-wide $30 \mathrm{~m}$ resolution DEM from SAR Interferometry in 11 days. Photogrammetric Week 99, Wichmann Verlag, Heidelberg.
Brown R.J., Brisco B., D'lorio M.A., Prevost C., Ryerson R.A. \& Singhroy V. (1996) RADARSAT Applications: Review of GlobeSAR Program, Canadian Journal of Remote Sensing, vol. 22(4), pp. 404-419.

Chandra M. \& Hounam D. (1998) Feasibility of a spaceborne P-band SAR for land surface imaging, Proceedings of EUSAR'98, Friedrichshafen, Germany, 1998.

Correia A. H. (2011) Metodologias e resultados preliminares do projeto radiografia da Amazônia. Anais $X V^{\circ}$ Simpósio Brasileiro de Sensoriamento Remoto, Curitiba, Brasil, INPE, p. 8083.

Droulers M. (1995) L'Amazonie, Editions NathanUniversité, collection Géographie d'aujourd'hui.

Dubois-Fernandez P. \& Koleck Th. (2014) Exploring the complementarities between $X$ and $P$ Band for vegetation characterisation. Proc. EUSAR 2014.

El Hage (2012) Etude de la qualité géomorphologique de modèles numériques de terrain issus de l'imagerie spatiale, thèse, CNAM.

Escobar I.P., Oliveira S.A.M., Lima S.P.S., Prado R.L. \& Ferreira A.T.A. (2005) Reprocessamento digital das imagens SLAR geradas pelos projetos RADAM e RADAMBRASIL - projeto RADAM-D. Anais XII Simpósio Brasileiro de Sensoriamento Remoto, Goiana, Brésil, INPE, pp. 4395-4397.

Farr T. \& Kobrick M. (2001) The Shuttle Radar Topography Mission, Eos Trans. American Geophys. Union, vol. 82, p.47.

Hayakawa E.H., Rossetti D.F. \& Valeriano M.M. (2010) Applying DEM-SRTM for reconstructing a late Quaternary paleodrainage in Amazonia, Earth and Planetary Science Letters, vol. 297, Issues 1-2, pp. 262-270.

INPE (2013) Metodologia para o Cálculo da Taxa Anual de Desmatamento na Amazônia Legal. http://www.obt.inpe.br/OBT/assuntos/programas/am azonia/prodes/pdfs/metodologia_taxaprodes.pdf (consulté en septembre 2017).

Kasser M. \& Egels Y. (Ed.) Digital Photogrammetry. London, Taylor \& Francis, 2002.

Leberl, F. W. (1990) Radargrammetric image processing. Artech House, Norwood, USA.

Le Toan T., Quegan S., Davidson M.W.J., Balzter H., Paillou P., Plummer S., Papathanassiou K., Rocca F., Saatchi S., Shugart H. \& Ulander L. (2011) The BIOMASS mission: mapping global forest biomass to better understand the terrestrial carbon cycle. Remote Sensing of Environment, 115 (11), pp. 2850-2860.

Le Toan T., Mermoz S., Bouvet A., Villard L. \& Polidori L. (2017) Monitoring of tropical forests using SAR data Application to the Amazon region Monitoring of tropical forests using SAR data - Application to the Amazon region. Anais $X V I I I^{\circ}$ Simpósio Brasileiro de Sensoriamento Remoto, Santos, Brésil, INPE, pp. 8076-8083.

Mantelli L.R., Rossetti D.F., Albuquerque P. G. \& Valeriano M.M. (2009) Applying SRTM digital elevation model to unravel quaternary drainage in forested areas of Northeastern Amazonia, Computers and Geosciences, vol. 35, Issue 12, pp. 2331-2337. 
Massonnet D. \& Rabaute Th (1993) Radar interferometry: limits and potential. IEEE Transactions on Geoscience and Remote Sensing, vol. 31, issue 2, pp. $455-464$.

Moreira J., Schwabisch M., Wimmer C., Rombach M. \& J. C. Mura (2001) Surface and ground topography determination in tropical rainforest areas using airborne interferometric SAR. Photogrammetric Week 01, Wichmann Verlag, Heidelberg.

Mougin E., Lopes A., Hery P., Marty G. Le Toan T., Fromard F. \& Rudant J.P. (1994) Multifrequency and multipolarization observations on mangrove forests of French Guyana during SAREX-92 experiment preliminary results. SAREX-92 Workshop Proceedings, ESA WPP-76, Paris, pp. 193-203.

Papathanassiou K. P., Cloude S.R. \& Reigber A. (2000) Single and multi-baseline polarimetric SAR interferometry over forested terrain. Proceedings of 3rd European SAR Conference EUSAR 2000, Munich, Germany, pp. 123-126.

Paquerault S. (1998) Restitution du relief à partir d'images radar par radarclinométrie, thèse, Télécom ParisTech.

Polidori L. (1991) Digital terrain models from radar images : a review. Proc. Int. Symp. on Radars and Lidars in Earth and Planetary Sciences, Cannes, pp. 141-146.

Polidori L. (1995) Réflexions sur la qualité des modèles numériques de terrain. Bulletin de la Société Française de Photogrammétrie et de Télédétection, N¹39, pp. 10-19.

Polidori L. (1997) Cartographie Radar, Gordon \& Breach, Amsterdam.

Polidori L. (2008) Remote sensing and coastal ecosystem monitoring in French Guiana : research and achievements over a decade. International Archives of the Photogrammetry, Remote Sensing and Spatial Information Sciences. Vol. XXXVII. Part B8. Beijing, pp. 627-531.

Polidori L., El Hage M. \& Valeriano M.M. (2014) Digital elevation model validation with no ground control ; application to the Topodata DEM in Brazil. Bol. Ciênc. Geod., sec. Artigos, Curitiba, v. 20, no 2, pp. 467-479.

Polidori L. \& Simonetto E. (2014) Effect of scale on the correlation between topography and canopy elevations in an airborne InSAR product over Amazonia. Procedia Technology 16 (2014), p. 180185, doi: 10.1016/j.protcy.2014.10.081.

Ramos F.L.G., Miranda F.P., Evsukoff A.G., Trouvé E. \& Galichet S. (2012) Fusion d'informations issues de la télédétection radar pour l'observation de déplacements dans la région de Manaus (Amazonie), RFPT, N 198-199, pp. 30-38.

Reigber A. \& Moreira A. (2000) First demonstration of airborne SAR tomography using multibaseline L-band data. IEEE Transactions on Geoscience and Remote Sensing, vol.38, N5, pp. 2142-2152.

Rodriguez E., Morris C.S. \& Belz J.E. (2006) A global assessment of the SRTM performance. Photogrammetric Engineering \& Remote Sensing, vol. 72, N³, pp. 249-260.
Rudant J.P. (1994) French Guyana through the clouds : first complete satellite coverage. ESA Earth Observation Quarterly, N44, pp. 1-6.

Silva E.A. (1998) Cartography and remote sensing $n$ the Amazon: the SIVAM project. IAPRS, vol. 32, Part 4 "GIS-Between Visions and Applications", pp. 580585.

Siqueira P., Hensley S., Shaffer S., Hess L., McGarragh G., Chapman B. \& Freeman A. (2000) A continentalscale mosaic of the Amazon basin using JERS-1 SAR imagery, IEEE Geosc. Rem. Sensing, vol. 38, Nº6, pp. 2638-2644.

Souza Filho P.W.M., Prost M.T. Miranda F.P., SalesM.E.C., Borges H.V., Costa F.R., Faria E. \& Nascimento Junior W.R. (2009) Environmental sensitivity index (ESI) mapping of oil spill in the Amazon coastal zone: the PIATAM Mar project, Revista Brasileira de Geofísica, vol.27 (Supl. 1), pp. 7-22.

Toutin Th \& Amaral S. (2000) Stereo Radarsat Data for Canopy Height in Brazilian Forests. Journal canadien de télédétection, vol. 26, Issue 3, pp. 189-199.

Valeriano M.M. \& Rossetti D.F. (2012) Topodata: Brazilian full coverage refinement of SRTM data. Applied Geography, vol. 32 N², pp. 300-309.

Van Roessel J. W. \& Godoy R. C. (1974) SLAR Mosaics for Project RADAM. Photogrametric Engineering, vol. 40, N5, pp. 583-595.

Vasseur A. (2002) Réalisation et analyse d'un modèle numérique de terrain radargrammétrique en forêt équatoriale, mémoire TFE, ESGT, Le Mans.

Wooding M. G., Zmuda A. D., \& Attema E. (1994) An overview of SAREX-92 data acquisition and analysis of the tropical forest environment, Proc. 2nd EuroLatin American Space Days, Buenos Aires, Argentina. Ed. Norman Longdon, ESA SP-363, pp.5768.

Zebker H.A. \& Goldstein R.M. (1986) Topographic mapping from interferometric SAR observations. J. Geophys. Res. 91 : 4993-9. 